Génét. Sél. Evol., 1983, 15 (4), 559-566

\title{
Tribune libre
}

\section{Lutte biologique et destruction du patrimoine génétique : le cas des mollusques gastéropodes pulmonés dans les territoires français du Pacifique}

\author{
Simon TILLIER et Bryan C. CLARKE * \\ Laboratoire de Biologie des Invertébrés marins et de Malacologie \\ 55, rue Buffon, F 75005 Paris \\ * Department of Genetics, University Park, Nottingham \\ NG7 2RD, Royaume-Uni
}

\begin{abstract}
Résumé
La faune des mollusques terrestres des îles du Pacifique constitue par sa richesse, sa diversité et son endémisme un matériel de tout premier choix pour les études génétiques, microévolutives et macroévolutives. Cette faune a été relativement peu affectée par le recul des forêts primaires lié aux activités humaines et par les introductions de mollusques phytophages, en particulier en Nouvelle-Calédonie. En Polynésie par contre, l'introduction ancienne et accidentelle de fourmis carnivores a fait disparaître presque totalement une radiation de plusieurs centaines d'espèces. Plus récemment, pour lutter contre l'escargot africain Achatina fulica Bowdich, les services ruraux ont introduit dans de nombreuses îles des escargots prédateurs d'autres Mollusques, dont Euglandina rosea (Férussac). Ces introductions n'ont pas d'effet apparent sur la dynamique des populations d'Achatines, mais détruisent très rapidement ce qui reste de la malacofaune primaire endémique des îles du Pacifique. Si rien n'est fait, un patrimoine génétique irremplaçable sera irrémédiablement perdu dans les prochaines décennies.
\end{abstract}

Mots clés : Patrimoine génétique, lutte biologique, extinction, mollusques terrestres, îles du Pacifique.

\section{Summary \\ Biological control and the destruction of our natural heritage : the fate of the land snails in the French Pacific territories}

The indigenous land snail species of the Pacific islands offer rich material for studies in genetics, microevolution and macroevolution. Until recently, this fauna has been relatively well preserved despite the destruction of primary forests by human activity and introduction of phytophagous land molluscs, particularly in New Caledonia. In Polynesia, however, the accidental introduction, some years ago, of carnivorous ants destroyed almost completely a radiation of several hundred species. Recent attempts by agricultural advisors to control the African giant snail, Achatina fulica Bowdich, led to the deliberate introduction of 
several land snail species, among them Euglandina rosea (Férussac), which prey on other molluscs. These predators have had no apparent effect in controlling Achatina, but they are rapidly destroying the primary endemic malacofauna. If nothing is done to protect this irreplaceable genetic resource, it will be irretrievably lost to science within decades. islands.

Key words : Genetic resource, biological control, extinction, land molluscs, Pacific

Depuis l'époque de Darwin, l'endémisme de la faune et de la flore des îles a fait de celles-ci un terrain de choix pour l'étude de l'évolution. Cet endémisme est grossièrement proportionnel à l'ancienneté et à l'isolement des îles, et en fait de véritables laboratoires naturels de l'évolution à différentes échelles :

- la présence d'ensembles de populations monophylétiques dont le degré d'isolement reproducteur est variable permet l'étude des phénomènes de spéciation (par exemple, LACK, 1947 ; Carson \& YoOn, 1982);

- la fermeture, plus ou moins complète, des écosystèmes insulaires vis-à-vis des immigrants permet d'établir et de tester les hypothèses globales sur l'exclusion compétitive, la diversité et la saturation des écosystèmes (théorie de l'équilibre des îles (Mc Arthur \& Wilson, 1963, 1967) et ses développements);

- la présence de groupes monophylétiques supragénériques permet d'envisager, dans la mesure où les rapports entre spéciation et conditions écologiques sont compris, l'étude et les tests de la théorie des radiations adaptatives (Simpson, 1944). Il apparaît que de telles radiations représentent le meilleur matériel accessible pour tester la puissance d'explication de concepts encore flous, comme ceux de la sélection d'espèces (STANLey, 1979) ou de l'exaptation (Gould \& VRBA, 1982).

La contrepartie des avantages que présentent les écosystèmes insulaires pour l'étude de l'évolution est leur fragilité, dont l'explication classique est leur imperméabilité, due à l'isolement, aux compétiteurs plus efficaces que les colonisateurs initiaux et leurs descendants.

Parmi les îles tropicales, connues depuis le siècle dernier comme les plus riches en taxons endémiques, les îles du Pacifique sont probablement celles qui ont été les mieux préservées au moins jusqu'au début de ce siècle. Sur ces îles la richesse et la diversité des mollusques terrestres, en relation avec leur faible mobilité, en font l'un des groupes zoologiques les plus intéressants pour l'étude des phénomènes évolutifs. Dans cet article, nous tentons d'une part d'évaluer les dégâts déjà commis involontairement par les activités humaines; d'autre part et surtout, de montrer grâce à l'exemple précis de l'introduction volontaire d'un escargot prédateur en Polynésie, qu'un programme de lutte biologique dont les effets n'ont pas été évalués a déjà détruit une partie importante d'un patrimoine génétique irremplaçable dont il menace l'ensemble.

\section{Malacofaune endémique de Polynésie}

L'âge estimé des îles françaises du Pacifique central (Marquises, Tuamotu, Société, Gambier, Australes) s'échelonne de 75-80 MA (Tuamotu) à moins d'un million d'années 
(Tahiti). Ce sont toutes des îles volcaniques, néoformées au milieu de l'océan. Leurs surfaces sont de l'ordre de quelques dizaines de kilomètres carrés, et l'altitude des îles hautes peut atteindre environ 2000 mètres (données synthétisées par SolEM, 1983). Leur faune de mollusques terrestres nous est connue principalement grâce aux récoltes du Bernice P. Bishop Museum (Hawaï) effectuées dans la première moitié du siècle. Cette faune comptait primairement plusieurs centaines d'espèces, toutes endémiques à différentes échelles, représentant une quinzaine de familles. Les représentants de trois familles seulement, les Endodontidae (SolEM, 1976), les Charopidae (SolEM, 1983) et les Partulidae, ont été étudiés récemment, en rapport direct avec les problèmes d'évolution énumérés plus haut.

Les radiations des Endodontoides (Endodontidae + Charopidae + Punctidae) comptent au total environ 300 espèces dans les îles du Pacifique, Nouvelle-Zélande et Nouvelle-Calédonie exceptées. Quelques dizaines d'espèces endémiques sont connues dans chaque archipel, quelquefois dans une seule île : 24 espèces à Rapa (Australes), 25 à Mangareva (Gambier). Dans la plupart des cas, il semble qu'un très petit nombre d'espèces de petite taille a colonisé chaque archipel ou chaque île. La spéciation et la radiation ont permis une diversification des tailles dans chaque groupe monophylétique. Globalement la diversité observée est souvent. plus élevée que ne le prévoit la théorie des îles (Solem, 1983).

Les phénomènes microévolutifs ont été part:culièrement étudiés chez les Partula (Partulidae) de Moorea (Iles de la Société ; $12 \mathrm{~km}$ de diamètre, 1207 mètres d'altitude, 1,2 MA). Les Partula se déplacent peu, se reproduisent vite et sont faciles à élever, sont ovovivipares et ont un polymorphisme génétique important qui se traduit aussi bien au niveau moléculaire qu'au niveau morphologique. Crampton (1932) et Crampton \& CoOKe (1953) ont décrit onze espèces, toutes endémiques. Deux d'entre elles, Partula solitaria Crampton et $P$. diaphana Crampton et Cooke, ont été rangées depuis dans le genre Samoana (Kondo, 1973). Deux autres, $P$. dendrö̈ca Crampton et $P$. olympia Crampton, sont des formes géographiques respectivement de $P$. suturalis Pfeiffer et de $P$. tohiveana Crampton (Clarke \& Murray, 1969 ; Murray \& Clarke, 1980). Sept espèces sont donc actuellement reconnues : Partula taeniata Mörch, $P$. exigua Crampton, $P$. suturalis Pfeiffer, $P$. tohiveana Crampton, $P$. aurantia Crampton, $P$. mooreana Hartman, et $\boldsymbol{P}$. mirabilis Crampton. On peut trouver dans la nature jusqu'à quatre espèces sympatriques isolées reproductivement et distinctes par leurs caractères génétiques, morphologiques, éthologiques et écologiques (Johnson, Murray \& Clarke, 1977 ; Murray \& Clarke, 1980 ; Murray, Johnson \& Clarke, 1982). Cependant le statut de ces espèces sur le plan biologique n'est pas aussi clair qu'il y paraît. En effet, il existe des zones d'hybridation entre couples d'espèces, de telle sorte qu'au moins potentiellement les gènes de n'importe laquelle de ces espèces peuvent être transmis à n'importe quelle autre.

\section{Malacofaune endémique de Nouvelle-Calédonie}

La Nouvelle-Calédonie, longue d'environ 500 kilomètres et large d'une cinquantaine de kilomètres, est beaucoup plus grande qu'aucune île du Pacifique central. Elle en est très différente géologiquement puisqu'elle représente, en schématisant, un morceau de la bordure orientale du Gondwana séparé de l'Australie orientale depuis le Trias (ca. $230 \mathrm{MA}$ ). Il est possible qu'elle ait été recouverte par deux transgressions 
marines, mais elle est restée constamment émergée depuis l'Oligocène (ca. $30 \mathrm{MA}$ ) (PARIS \& Lille, 1977). L'interaction de son relief, qui s'élève jusqu'à 1600 mètres d'altitude, avec les masses d'air venant du nord-est détermine des variations climatiques brutales (de 6 mètres de pluie par an à moins d'un mètre sur des distances de l'ordre de la dizaine de kilomètres). Il en résulte une mosaïque de microclimats à laquelle correspond une mosaïque d'environnements.

Environ 110 espèces de mollusques terrestres, toutes endémiques, y ont été décrites au siècle dernier (Solem, 1961). Les récoltes effectuées depuis 1977, principalement par Bouchet et Tillier, permettent d'estimer le nombre total d'espèces endémiques non éteintes entre 300 et 400 . La plupart de ces espèces sont endémiques d'une petite partie de l'île. Une faible proportion, de l'ordre de 10 p. 100, représente des immigrants récents à l'échelle géologique, dont les ancêtres sont arrivés grâce à la formation de l'arc indomalais (Helicarionidae, Enidae, Diplommatinidae; SolEM, 1959). Le reste de la malacofaune montre clairement des affinités gondwaniennes (Australie, Nouvelle-Zélande, Amérique du Sud, Afrique du Sud) et est le résultat de radiations à partir d'un petit nombre d'espèces isolées anciennement (au moins depuis l'Oligocène, au plus depuis le Trias). Dans ce groupe, au moins 80 p. 100 des espèces représentent probablement une radiation monophylétique des Charopidae, et 10 p. 100 appartiennent à un groupe ici prédateur des Charopidae, les Rhytididae (TILliER, non publié).

Les espèces révisées à ce jour montrent des variations clinales extrêmement importantes qui sont corrélées soit avec la présence d'espèces congénériques sympatriques, soit avec les variations des conditions d'environnement (TILliER, 1981 a ; Chérel-Mora, 1983). Ces données permettent d'espérer qu'on pourra corréler les facteurs de la variabilité intraspécifique et intragénérique, la composition des guildes et les relations phylétiques des taxons, et étudier ainsi les modalités des radiations adaptatives.

\section{Extinctions et introductions antérieures à 1967}

La colonisation des îles du Pacifique par les Mélanésiens et les Polynésiens, puis par les Européens a provoqué un recul de la forêt primaire et la disparition de la faune qui y était associée. Il est difficile d'apprécier l'âge et l'étendue des destructions par l'homme, mais il est important de savoir que ces destructions ont affecté pour l'esentiel les forêts de basse altitude et les espèces de grande taille. Les habitats détruits étaient moins diversifiés qu'en altitude, de telle sorte que le nombre d'extinctions directement liées au recul de la forêt est sans doute assez faible : le plus souvent, les espèces endémiques de taille modérée ont pu (Nouvelle-Calédonie) ou auraient pu (Polynésie) subsister dans les lambeaux de forêt de basse altitude qu'on trouve encore dans des endroits peu accessibles ou le long des cours d'eau. Dans l'ensemble le recul de la forêt a été plus important en Polynésie qu'en Nouvelle Calédonie (cartographiée par l'O.R.S.T.O.M., 1981).

L'homme a introduit, avec les végétaux cultivés, une trentaine d'espèces de mollusques terrestres dont la plupart existent maintenant dans toute la zone intertropicale («tropical tramps»; Solem, 1964). Ces espèces sont en majorité de petite taille et ne 
constituent généralement pas une gêne pour l'agriculture. Nous ne disposons pas de données précises sur leur pénétration dans les milieux primaires de Polynésie, mais il est certain qu'elles sont strictement inféodées aux milieux secondaires en NouvelleCalédonie (TILlier, non publié). Il n'y a pas de raison de supposer que ces espèces ont été ou sont des compétiteurs de la malacofaune primaire.

Malheureusement les Européens ont aussi introduit involontairement des fourmis carnivores dont l'effet a été dramatique en Polynésie. Solem (1976, 1983) estime qu'elles ont détruit presque entièrement la faune d'Endodontidae de Polynésie : une radiation de plusieurs centaines d'espèces a disparu entre 1900 et 1960 dans l'indifférence générale, et est définitivement inaccessible aux études génétiques et microévolutives.

\section{Introduction d'Achatina fulica}

L'escargot géant africain, Achatina fulica Bowdich, a été introduit peu à peu depuis le XVIII' siècle, à partir d'Afrique orientale, dans les pays de l'Océan indien et du Pacifique. C'est un fléau majeur pour la polyculture en milieu tropical (MEAD, 1961, 1979). Il a été introduit volontairement par des particuliers à Tahiti en 1967, et en Nouvelle-Calédonie en 1972 [soit pour des tentatives d'élevage, soit dans un but décoratif (!)], puis dans d'autres territoires français du Pacifique. Sa démographie est partout semblable. Les populations connaissent d'abord une phase d'expansion explosive, au cours de laquelle les densités atteignent des valeurs extrêmement élevées (environ $400 \mathrm{~kg} / \mathrm{ha}$ en moyenne et 20000 ha envahis en Nouvelle-Calédonie en 1981 ; Tillier, 1981 b). Cette phase explosive, qui a culminé à Tahiti en 1979 et qui n'était pas terminée en Nouvelle-Calédonie en 1981, est suivie par une phase de déclin dont les causes sont inconnues : épizootie, prédation par des planaires (MEAD, 1979)... Les populations causent encore des dégâts dans les champs, mais l'importance du problème est alors moindre. En Nouvelle-Calédonie, les Achatines sont inféodées aux milieux secondarisés (au moins jusqu'en 1981; Tillier, 1981 b). En Polynésie, il semble qu'elles colonisent partiellement les milieux primaires, au moins le long des cours d'eau, mais la densité des populations et la taille des individus restent généralement faibles (PoInTIER, com. pers.).

Par leur taille, leur densité et les dégâts qu'elles causent aux cultures au cours de la phase d'expansion des populations, les Achatines ont constitué un problème politicoéconomique important dans les Territoires français du Pacifique. Le problème a été amplifié par la crainte de la méningite éosinophile dont les Achatines sont vectrices. Cette crainte apparaît très exagérée, puisque :

- la méningite éosinophile est transmise par plusieurs espèces introduites très communes dans toute la zone intertropicale depuis plus de cent ans (CHENG \& AliCATA, 1965) ;

- le nombre de cas de méningite enregistrés à Nouméa a très nettement diminué depuis une dizaine d'années, alors même que les populations d'Achatines étaient en pleine expansion (Bouchet, enquête non publiée auprès des médecins de Nouméa). 


\section{Introduction d'Euglandina rosea et ses conséquences}

Euglandina rosea (Férussac) est un escargot Oleacinidae prédateur d'autres escargots. Depuis les années 1930, il a été utilisé pour des essais de «lutte biologique» contre Achatina fulica. Bien qu'il puisse se nourrir d'Achatines, son efficacité en tant que moyen de contrôle démographique de cette espèce n'a jamais été démontrée (MEAD, 1979). Cependant son introduction a été recommandée par de nombreux services ruraux et par la Commission du Pacifique Sud.

Mis en demeure par la pression de l'opinion publique de mettre en auvre des moyens de lutte contre l'Achatine, les services ruraux ont introduit $E$. rosea dans une plantation de Moorea en 1977, et à Nouméa et à Ouégoa (Nouvelle-Calédonie) en 1978. Il semble que la population de Ouégoa avait disparu en 1981, mais Euglandina a été récoltée à Nouméa en 1980. A Moorea, elle a peu à peu envahi les forêts tout autour de la plantation où elle avait été introduite avec une vitesse d'expansion d'environ 1,2 km par an. A cette vitesse, elle aura envahi toute l'île en 1986-1987.

Le déclin des populations d'Achatines à Moorea depuis 1978-1979 ne peut pas être attribué à l'introduction de E. rosea : on trouve encore des Achatines vivantes sympatriques avec Euglandina, et le déclin des populations d'Achatines a été observé dans des vallées où Euglandina est absente (par exemple les vallées d'Aareo et de Vaihiiaiia). De plus, un déclin semblable a été observé sur l'île de Huahine, où $E$. rosea n'a pas été introduite (Pointier \& BLANC, 1982).

Il n'y a malheureusement aucun doute à propos de l'effet de l'introduction d'E. rosea sur les populations de Partula, qui sont totalement détruites par le prédateur (placées dans une boîte avec une E. rosea, quatre Partula ont été dévorées en 24 heures). En 1982, Clarke, Murray et Johnson n'ont pu trouver aucune Partula vivante dans des localités envahies par E. rosea où les Partula abondaient en 1967 et en 1980. Une espèce, Partula aurantia, est maintenant totalement éteinte dans la nature et n'existe plus qu'en élevage au laboratoire. Une sous-espèce, $P$. taeniata striolata, est totalement éteinte. Trois espèces $(P$. suturalis, $P$. tohiveana et $P$. taeniata) ont totalement disparu du nord-ouest de l'île mais survivent provisoirement ailleurs. Seuls quelques individus de $P$. exigua et quelques hybrides exigua $\times$ taeniata ont été trouvés vivants dans la zone envahie par $E$. rosea. Nous ignorons pourquoi ils ont survécu, et si cette survie est durable.

\section{Menaces à moyen et long terme}

Euglandina rosea a été introduite en 1974 à Tahiti, où elle occupait en 1982 toute la vallée de Temarua et le plateau de Taravao. Dans la zone envahie aucune Partula ne semble avoir survécu (Pointrier \& Blanc, 1982). Par ailleurs il semble que $E$. rosea menace non seulement les autres Partulidae, mais l'ensemble de la malacofaune endémique; en tout cas, l'Helicarionidae autrefois commun Trochomorpha pallens Pease semble aussi avoir disparu des zones envahies par E. rosea. Si la vitesse d'expansion du prédateur est la même partout, on peut espérer que la faune de Tahiti survivra plus longtemps que celle de Moorea, et qu'il faudra plus de cent ans pour que la malacofaune endémique de Nouvelle-Calédonie disparaisse totalement. 
En Nouvelle-Calédonie cependant, certaines espèces de Charopidae sont endémiques de la chaîne du Mont Koghi, tout près de Nouméa, et sont d'ores et déjà directement menacées.

Deux autres espèces d'escargots prédateurs qui se nourrissent des œufs d'autres mollusques, Gonaxis quadrilateralis (Preston) et G. kibwenziensis (E.A. Smith), ont été introduites dans de nombreuses îles du Pacifique occidental et de l'Océan indien, dont Guam et Saipan où la situation est probablement déjà pire que dans les territoires français du Pacifique (MEAD, 1979). Connaissant la vitesse d'expansion d'Achatina fulica et l'obstination des services ruraux, on peut affirmer que ce qui reste de l'extraordinaire malacofaune de toutes ces îles est directement menacé. On peut estimer qu'au moins une centaine d'espèces ont déjà disparu, et que plusieurs centaines d'autres sont condamnées.

\section{Conclusion}

Nous ne connaissons malheureusement aucun moyen d'arrêter l'expansion d'Euglandina rosea et des autres prédateurs potentiels d'Achatina fulica introduits. Bien que l'inefficacité de ces introductions pour la lutte contre les Achatines et leur danger pour la faune endémique soient maintenant bien établis, nous ne pouvons qu'être pessimistes quant à l'arrêt des introductions. En effet, les fortes mises en garde par P. Bouchet auprès des services ruraux de Nouvelle-Calédonie n'ont pas empêché une seconde introduction; il semble aussi que son action auprès de la Commission du Pacifique Sud soit restée sans effet. De la même façon, il ne semble pas que les mises en garde de J.B. Burch (en 1970) et de Pointier et Blanc (en 1982) auprès des services ruraux de Polynésie modifient leur politique catastrophique d'introduction d'E. rosea.

Nos moyens d'action sont dérisoires comparés à l'ampleur de la menace qui pèse sur les ressources génétiques que représentent les mollusques terrestres des îles du Pacifique. Nous pouvons :

- soit étudier aussi rapidement et complètement que possible les phénomènes évolutifs et la génétique des espèces menacées, en particulier lorsque la faune est encore presque totalement préservée comme en Nouvelle-Calédonie ;

- soit, dans le cas de groupes restreints dont l'élevage est possible, tenter de maintenir des populations en laboratoire. Cette expérience est actuellement tentée avec les Partula de Moorea à Nottingham, Jersey et Londres grâce à l'aide du Jersey Wildlife Preservation Trust et de la Zoological Society of London, ainsi qu'à Perth (Australie) et à Charlottesville (U.S.A.), grâce aux efforts de J.J. Muray et de M.S. Johnson.

En tout état de cause et quoi que nous fassions, nous ne pouvons que tenter de limiter les dégâts avant qu'un patrimoine génétique irremplaçable ne soit perdu. Nous espérons que cet exemple rendra les services officiels, responsables des introductions actuellement les plus dangereuses pour le patrimoine génétique, plus prudents dans leurs tentatives de lutte biologique. Il est indispensable que les recherches sur les moyens de lutte biologique prennent en compte non seulement l'impact sur les espèces nuisibles, mais aussi et peut-être surtout l'impact sur les espèces non nuisibles. 


\section{Références bibliographiques}

Carson H.L., Yoon J.S., 1982. Genetics and evolution of Hawaiian Drosophila. In : Ashburner M., CARSON H.L., ThOMPSON J.N. (ed.), The genetics and biology of Drosophila, vol. 3 b, 207-344. Academic Press, London.

Cheng T.C., Alicata J.E., 1965. On the modes of infection of Achatina fulica by the larvae of Angiostrongylus cantonensis. Malacologia, 2, 267-274.

Cherel-Mora C., 1983. Variation géographique et taxonomie des Placostylus (Gastéropodes pulmonés stylommatophores) en Nouvelle-Calédonie. Thèse de $3^{\mathrm{e}}$ cycle, Université Paris VII, $103 \mathrm{p}$.

Clarke B., Murray J., 1969. Ecological genetics and speciation in land snails of the genus Partula. Biol. J. Linn. Soc. Lond., 1, 31-42.

Crampton H.E., 1932. Studies in the variation, distribution and evolution of the genus Partula. The species inhabiting Moorea. Publ. Carnegie Inst., 410, 1-335.

Crampton H.E., Cooke C.M., 1953. New species of Partula from southeastern Polynesia. Occ. Pap. Bernice Pauahi Bishop Mus., 21, 135-159.

Gould S.J., VRBA E., 1982. Exaptation : a missing term in the science of form. Paleobiology, 8, 4-15.

Kondo Y., 1973. Samoana of the Society islands (Pulmonata-Partulidae). Malacol. Rev., 6, 19-33.

LACK D., Darwin's finches. 204 p. Harper Bros., New York.

MaC ARThur R.H., Wilson E.O., 1963. An equilibrium theory of insular zoogeography. Evolution, 17, 373-387.

MaC ARThur R.H., Wilson E.O., 1967. The theory of island Biogeography. 203 p. Princeton University Press, Princeton.

MEAD A.R., 1961. The giant African snail : a problem in economic malacology. 255. p., University of Chicago Press, Chicago.

Mead A.R., 1979. Economic malacology with particular reference to Achatina fulica. In : Fretter V., Peake J. (ed.), Pulmonates, vol. 2 B, 150 p., Academic Press, London.

Murray J., Clarke B., 1980. The genus Partula in Moorea : speciation in progress. Proc. R. Soc., B, 211, 83-117.

Murray J., Johnson M.S., Clarke B., 1982. Microhabitat differences among genetically similar species of Partula. Evolution, 36, 316-325.

OrSTOM, 1981. Atlas de la Nouvelle-Calédonie. 53 pl., O.R.S.T.O.M., Paris.

Paris J.P., Lille R., 1977. La Nouvelle-Calédonie du Permien au Miocène. Bull. Rech. Geol. Min., $4^{\mathrm{e}}$ série, 1, 79-95.

Pointier J.P., Blanc C., 1982. Achatina fulica dans les iles de la Société. Rapport, Muséum national d'Histoire naturelle, Antenne de Tahiti, Papeete.

Simpson G.G., 1944. Tempo and Mode in Evolution. 237 p., Columbia University Press, New York.

Solem A., 1959. Systematics and zoogeography of the Land and Freshwater Mollusca of the New Hebrides, Fieldiana Zool., 43, $359 \mathrm{p}$.

SOLEM A., 1961. New Caledonian land and freshwater snails : an annotated checklist. Fieldiana Zool., 41, 413-501.

Solem A. 1964. New Records of New Caledonian Nonmarine Mollusks and an Analysis of the Introduced Mollusks. Pac. Sci., 18, 130-137.

Solem A., 1976. Endodontoid land snails from Pacific islands. Part I. Family Endodontidae. 508 p., Field Museum of natural History, Chicago.

Solem A., 1983. Endodontoid land snails from Pacific islands. Part II. Families Punctidae and Charopidae. 336 p., Field Museum of Natural History, Chicago.

Stanley S.M., 1979. Macroevolution. Pattern and Process. 332 p., Freeman and Co, San Francisco.

Tillier S., 1981 a. Clines, Convergence and Character Displacement in New Caledonian Diplommatinids (Land Prosobranchs). Malacologia, 21, 177-208.

TILlier S., 1981 b. L'escargot phytophage Achatina fulica en Nouvelle-Calédonie. Répartition, stocks, possibilités de lutte et d'exploitation. Rapport, Muséum national d'Histoire naturelle, Paris et Service d'Action et de Développement économique, Nouméa. 